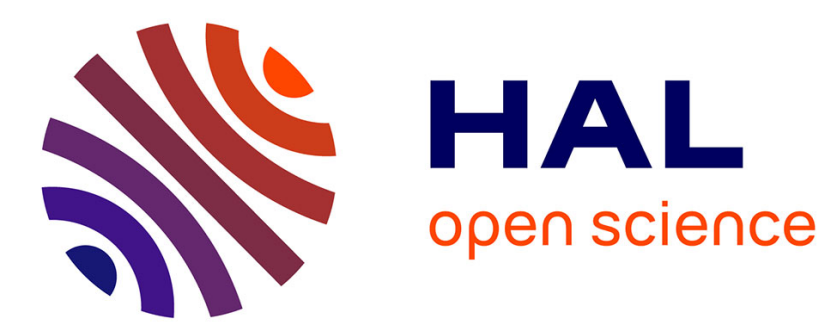

\title{
Multisensory integration in spatial orientation
}

Alain Berthoz, Isabelle Viaud-Delmon

\section{To cite this version:}

Alain Berthoz, Isabelle Viaud-Delmon. Multisensory integration in spatial orientation. Current Opinion in Neurobiology, 1999, 9 (6), pp.708-712. 10.1016/S0959-4388(99)00041-0 . hal-03411667

\section{HAL Id: hal-03411667 \\ https://hal.science/hal-03411667}

Submitted on 8 Nov 2021

HAL is a multi-disciplinary open access archive for the deposit and dissemination of scientific research documents, whether they are published or not. The documents may come from teaching and research institutions in France or abroad, or from public or private research centers.
L'archive ouverte pluridisciplinaire HAL, est destinée au dépôt et à la diffusion de documents scientifiques de niveau recherche, publiés ou non, émanant des établissements d'enseignement et de recherche français ou étrangers, des laboratoires publics ou privés. 


\section{Multisensory integration in spatial orientation Alain Berthoz and Isabelle Viaud-Delmon}

Recent psychophysical studies on normal subjects, as well as brain imaging studies, have revised the concepts concerning the mechanisms underlying spatial orientation during navigation tasks. The emphasis has been put on internal models that allow the prediction of a planned trajectory and are essential in the steering of locomotion. Cognitive factors such as strategies and emotional parameters are now starting to be included in the research on spatial orientation. It is obvious that important individual and gender differences exist in the brain operations underlying spatial orientation in humans, which may help to understand the construction of a coherent perception and the organic neural disorders related to the internal representation of space.

Laboratoire de Physiologie de la Perception et de l'Action, Collège de France CNRS UMR 9950, 11 place Marcelin Berthelot, 75005 Paris, France

Current Opinion in Neurobiology 1999, 9:708-712

\section{Introduction}

The control of spatial orientation during navigational tasks and locomotion requires a dynamic updating of the representation of the relations between the body and the environment. This depends upon central integration of current multisensory information but also on the comparison of sensory signals with planned trajectories, the body schema and past memories. Three main sensory modalities are involved in these processes: vision, the vestibular system and proprioception. In addition, efferent copies from motor signals also contribute to the updating of spatial representations during navigation. The brain mechanisms underlying spatial orientation have, in the past, mainly been studied in static conditions. We shall review here some recent studies concerning the brain mechanisms that are used to dynamically update, or memorise, routes or travelled paths. This review will also emphasise a neglected dimension in the study of spatial orientation: the role of emotion in the underlying processes. This role is examined by recent studies concerning the relationship between spatial orientation and anxiety disorders.

\section{Vestibular motion perception and memory during navigation}

Despite a variety of models of sensory interaction $\left[1,2,3^{*}, 4^{* *}\right]$, the neuronal mechanisms of high-level interaction between vestibular and other sources of information remains unclear. In addition, motion perception also depends heavily upon internal models and not only on input-output transformations, but there is no general theory that can explain how the different afferent signals are combined in a unique coherent perception during navigation.

Recently, brain imaging techniques have improved the anatomical identification of the projection areas of vestibular, visual and kinaesthetic signals to the multimodal integration areas of the cerebral cortex involved in spatial orientation and memory in humans $\left[5^{\circ}, 6\right]$, and in the determination and perception of the mid-sagittal egocentric body reference [ $\left.7^{\circ}\right]$. These data, together with the data obtained in the primate cortex concerning the cortical systems dealing with head-in-space movement (see $\left[8^{* *}\right]$ ), contribute to the knowledge of the neural structures involved in space perception but they provide little information concerning the neural processes involved. Novel psychophysical methods in humans, however, have allowed the study of both canal and otolith organs during tasks involving the perception and memory of bi-dimensional motion during navigation [9]. Mobile robots can be used to passively displace human subjects while visual stimuli can be provided by virtual reality techniques. Together, these recent developments have given rise to new tools for the study of visual-vestibular interactions during combined rotations and translations. They have also allowed the experimental testing of new hypotheses concerning the type of information that is stored in spatial memory during navigation. Such experiments have suggested that the brain stores dynamic patterns of motion, rather than just position on cartesian-like cognitive maps [10]. These, together with observations of deficits in vestibular memory in patients with cortical lesions, and positron emisson tomography (PET) studies of the brain areas involved in the memory of travelled routes, have led to the formulation of the concept of 'topokinetic memory' [11], (i.e. the dynamic memory of the movements and associated landmarks and views during locomotion).

New methods of recording head and body movements during blind locomotion have also allowed the study of the spatial memory mechanisms underlying the steering of locomotion. In human subjects executing a locomotor turn - even in darkness - gaze direction leads the trunk and feet. This suggests that the steering is performed through predictive processes, probably involving complex interactions between kinesthetic information and internal models of the planned trajectory $\left[12^{\circ}\right]$. This predictive ability is not present in young infants and develops during the first few years [13 ${ }^{\circ}$. It has also been proposed that there is a dissociation between the coding of distance and direction for the steering of locomotion [14 $\left.{ }^{\circ}\right]$.

\section{Spatial orientation and emotion}

All the above results indicate that spatial orientation during navigation is dependent upon higher cognitive 
processes involving the limbic and cortical areas related to spatial memory. However, another dimension has also recently been revealed to play a role in these mechanisms. There is a remarkable overlap between the brain structures involved in spatial orientation and spatial memory, and those involved in emotion (see [15,16]). Spatial disorientation is a complaint reported by many patients in psychiatry. Evidence of dysfunction of spatial orientation has been found in agoraphobics [17]. During navigation in a complex environment, panic-agoraphobic patients became lost more often and utilised fewer navigation points. Moreover, the maps drawn afterwards by these patients were inaccurate [18].

The experience of vertigo and dizziness is a common symptom in several psychiatric disorders. Dizziness is also one of the key symptoms of panic. Because vestibular system dysfunction leads to dizziness and disorientation, it has been hypothesised that a peripheral vestibular abnormality could explain the presence of certain symptoms related to space perception in anxiety disorders [19]. Despite a strong association between dizziness and anxiety $\left[20^{\bullet}\right]$, however, it is difficult to demonstrate a cause-andeffect relationship between pathological anxiety and vestibular system dysfunction. There is a growing literature on vestibular abnormalities in anxiety disorders, in particular agoraphobia and panic disorder (see $\left[21^{\circ}, 22^{\circ}\right]$ for reviews). Numerous studies have investigated a potential link between anxiety and the vestibular system, but few of them have addressed the specific topic of spatial representation. In particular, the problem of the construction of a coherent perception of space has not been addressed in these studies.

Dizziness is characterised by a marked distortion of self-world relations and reflects a discrepancy between internal sensation and external reality. Spatial disorientation, as well as dizziness, can be due to a peripheral problem in any of the sensory modalities; or, it amy be due to a central problem, involving not one particular sensory modality but rather the integration and weighting of the different modalities and their relation with memory. Complaints concerning spatial disorientation in psychiatry have seldom been considered as a possible manifestation of a distorted multisensory integrative ability. Several kinds of sensory mismatches are encountered in everyday life but despite this, the central nervous system usually manages to update the internal representation of the body in the surrounding space. Such sensory conflicts may occur between any of the vestibular, visual or somatosensory systems. For example, passive transports generate sensorimotor mismatch because of the absence of motor feedback. In some cases, a mismatch among simultaneous sensory information may elicit an erroneous perception of the body in space, resulting in a vertigo syndrome. Vertigo may thus be induced by physiological external stimulation. However, when no physical explanation is found to explain the symptom of vertigo, it is common to label it as 'psychogenic'. We think it is necessary to go beyond such a dichotomy between 'physiological' and 'psychogenic' vertigo in order to reach a better comprehension of the mechanisms of spatial orientation.

\section{Space phobia}

A number of phobias that are acquired by dizziness-prone patients have been described. They often involve avoidance of stimulus situations having certain visual characteristics.

Space phobia [23] is characterised by fear of the absence of visual orienting cues and fear of falling, which is unlike the fear of public places found in agoraphobia. Space and motion discomfort [24] occurs in situations characterised by inadequate visual or kinaesthetic information for normal spatial orientation, and may contribute to promoting agoraphobic avoidance in patients with vestibular dysfunction. Postural phobic vertigo [25] refers to dizziness and subjective disturbance of balance while standing or walking despite normal clinical balance tests. Perceptual stimuli such as a bridge, a staircase, an empty room, a street or a social situation (e.g. a crowd, restaurant or concert) are provoking factors. The Motorist Disorientation Syndrome [26] refers to a difficulty when driving over the crests of hills and on open, featureless roads.

In all these syndromes, a false sense of orientation arises either from inappropriate signals from distorted vestibular canal and otolith organs, in the case of a vestibular dysfunction, or from a disordered central interpretation of sensory information. Symptoms of dizziness are mainly precipitated by certain visual surroundings. Some vestibular patients are sensitive to particular visual environments (e.g. supermarket aisle, crest of a hill when driving). Idiosyncratic visual and vestibular factors may play a part in the outcome of any peripheral of central lesion that causes loss of balance and spatial orientation, underlining the necessity to study the variation of the tolerance to visual and vestibular stimuli among normal people. Bronstein [27] proposed that visual vertigo may occur specifically in patients with balance disorders who show high reliance on visual channels for postural control and spatial orientation.

Agoraphobic patients demonstrate an abnormal destabilisation when maintaining upright posture under sensory conflicting conditions [28,29]. It has been hypothesised that dizziness symptomatology could be linked to a strategy for maintaining balance or orientation that relies on a dominant contribution of vision. Even though a recent study [30] attributed a stronger role of proprioceptive cues in the maintenance of balance in agoraphobic patients, the results of this study illustrated once more the possible role of a higher process involving multisensorial integration in anxiety disorders. It is indeed striking to note that an altered visual-vestibular interaction has often been observed in anxious patients. In addition, we have recently obtained evidence in anxious subjects of a lack of anticipatory oculomotor behaviour during passive rotations in darkness, which may reveal a physiological manifestation of 
a differing orientation strategy [31] in these persons compared with nonanxious subjects.

\section{Gender and sensory integration}

There are several sources of individual differences in space orientation, the most recognised of which is gender. Differences in space orientation between males and females have often been approached in terms of differing spatial abilities, but some studies indicate that gender differences in sensory integration could also exist.

Evidence for gender differences in circular vection (i.e. the illusion of self-motion when viewing moving visual surrounds) exists [32], and could be related to the greater susceptibility of females to motion sickness [33]. The 'mal de débarquement' syndrome (the sensation of rocking and swaying that persists after sea travel) also appears to be more common among females. The etiology of this syndrome is unknown, but it has been thought to result from an inability of the brain to integrate and adjust to new spatial surroundings [34]. In an experiment on sensory adaptation, female subjects were found to recalibrate their vestibular perception to a lesser extent after exposure to conflicting visual-vestibular stimuli $\left[35^{\circ}\right]$. All these findings consistently suggest gender differences in the central processes dealing with visual-vestibular interaction and internal strategies of spatial orientation and spatial memory.

We cannot exclude the idea that the observed differences in sensory integration in males and females are related to the differences observed in the ability to maintain a sense of self-position in relation to the global shape of the environment. Several studies have demonstrated that females use distal cues for spatial orientation in a different way to males (see [36]). While it has already been proposed that gender differences in the structure of the hippocampus, caused by the effects of sex hormones [37], could represent a neural correlate of the difference in spatial ability, the neural substrates of the difference in sensory integration are unknown. Further research using magnetic and metabolic brain imagery methods will be necessary if we are to understand the underlying differences between human males and females in the processing of spatial information.

\section{Conclusions and future directions}

It is now necessary to obtain more information concerning the neural basis of these higher cognitive processes. Brain imaging will certainly be useful, but we believe that recording of neurones in freely-moving monkeys during navigation tasks will also be absolutely necessary. The recent discoveries of Rolls et al. [38] concerning the mechanisms by which the monkey hippocampus may code the relationship between the animal and its surrounding space are examples of the new approaches that may be followed fruitfully. It will also be important in the future to study the individual strategies that are used by subjects of the same, and of differing sexes, to solve spatial problems. For example, the simple measurement of eye movements during a rotation memory test suggests that different subjects can use either allocentric or egocentric strategies [39*]. Moreover, during a locomotor task the brain can either update the location and orientation of objects in a continuous manner or delay the updating until the end of the locomotion, performing only an egocentric path integration during the travelled route [40].

More investigations are needed to analyse different aspects of multisensory integration in anxious patients in tasks implying perception as well as motor control. In particular, the roles of the hippocampus, parietal cortex and frontal cortex in the mechanisms that allow steering of locomotion during navigation tasks should be investigated. More generally, it is necessary to design brain imaging experiments in humans using paradigms in which subjects recall actual, actively-performed navigation tasks. Some attempts in this direction have been made [41] and should be continued.

\section{Acknowledgements}

This review was supported by a grant from SmithKline Beecham, the Human Frontier Research program and the European laboratory Laboratoire Européan des Neurosciences de l'Action. IV-D was supported by Fondation de la Recherche Médicale. Particular thanks to Joe McIntyre for his suggestions and comments on the manuscript.

\section{References and recommended reading}

Papers of particular interest, published within the annual period of review, have been highlighted as:

- of special interest

$\bullet$ of outstanding interest

1. Borah J, Young, LR, Curry RE: Optimal estimator model for human spatial orientation. Ann NY Acad Sci 1988, 545:51-73.

2. Droulez J, Darlot C: The geometric and dynamic implications of the coherence constraints in three dimensional sensorimotor coordinates. In Attention and Performance XIII. Edited by Jeannerod M. New Jersey: Laurence Erlbaum Associates; 1989:495-526.

3. Merfeld DM, Zupan L, Peterka RJ: Humans use internal models to -. estimate gravity and linear acceleration. Nature 1999, 398:615-618. One of the most important unsolved questions concerning spatial orientation is the mechanism by which the brain separates the acceleration of gravity and the linear acceleration of the head during translations. Several authors have suggested that this is performed by a separation of the inertial and the gravitational components of head movements through a neural mechanism of filtering in the brain stem. Low pass filtering would yield the component due to gravity and high pass filtering to linear acceleration. This paper reports experiments on human subjects submitted to a tilt after having been rotated around an earth-vertical axis. Eye movement recordings provide evidence that, contrary to the above hypothesis, the brain solves the problem through an internal model of the difference between gravity and acceleration.

4. Mergner T, Rosemeier T: Interaction of vestibular, somatosensory

- $\quad$ and visual signals for postural control and motion perception under terrestrial and microgravity conditions - a conceptual model. Brain Res Brain Res Rev 1998, 28:118-135.

This model of multisensory interaction differs from classical models published so far in that it supposes that instead of combining directly, visual and vestibular signals are referred to a common reference provided by proprioceptive cues from the postural base, which is provided by the lower limbs in the case of a standing or a sitting subject. This model also contains a number of original features which are supported by the extensive exploration made by Mergner and his colleagues on the higher cognitive mechanisms that control multisensory integration of spatial cues.

5. Lobel E, Kleine JF, Bihan DL, Leroy-Willig A, Berthoz A: Functional

- MRI of galvanic vestibular stimulation. J Neurophysiol 1998 80:2699-2709.

This is a functional magnetic resonance imaging study of the cortical areas in humans activated by galvanic vestibular stimulation. The following areas were activated: the temporo-parietal junction, the central sulcus and the 
intra-parietal sulcus. These areas could be the equivalent of areas PIVC, $3 \mathrm{aV}$, and $2 \mathrm{v}$ in the monkey found by Grüsser and already described in the positron emission tomography literature. A new area was found to be activated in the premotor region of the frontal lobe.

6. Brandt T, Bartenstein P, Janek A, Dieterich M: Reciprocal inhibitory visual-vestibular interaction. Visual motion stimulation deactivates the parieto-insular vestibular cortex. Brain 1998, 121:1749-1758.

7. Vallar G, Lobel E, Galati G, Berthoz A, Pizzamiglio L, Le Bihan D: A

- fronto-parietal system for computing the egocentric spatial frame of reference in humans. Exp Brain Res 1999, 124:281-286.

The brain areas involved in the processing of the egocentric body midline were studied by functional magnetic resonance imaging in human subjects. Subjects were asked to indicate with a button when a visual target moving on a screen was either passing through the mid-sagittal plane of the body or through the two extremities of a linear horizontal trajectory. A parieto-frontal system was found to characterise the specific processing of the mid-sagittal plane. The location of the two areas is akin to the right posterior-inferior parietal cortex, in which lesions induce the neurological syndrome known as neglect.

\section{Guldin WO, Grüsser OJ: Is there a vestibular cortex? Trends}

-. Neurosci 1998, 21:254-259.

The authors review the data obtained in the primate cortex concerning the cortical vestibular representation. The vestibular cortical system considered in the review includes the parietal insular vestibular cortex, areas $3 \mathrm{a}, 2 \mathrm{v}, 7$, $6 \mathrm{v}$, and the visual posterior sylvian area. It is suggested that these different areas process information concerning head-in-space and head-on-body, and that the vestibular cortical system might be able to have direct influence on vestibular reflexes through feedback down to the vestibular brainstem nuclei.

9. Ivanenko YP, Grasso R, Israël I, Berthoz A: The contribution of otoliths and semicircular canals to the perception of twodimensional passive whole-body motion in humans. J Physiol (Lond) 1997, 502:223-233.

10. Berthoz A, Israël I, Georges François P, Grasso R, Tzuzuku T: Spatial memory of body lineardisplacement: what is being stored? Science 1995, 269:95-98.

11. Berthoz A: Parietal and hippocampal contribution to topokinetic and topographic memory. Philos Trans $R$ Soc Lond B Biol Sci 1997, 352:1437-1448.

12. Grasso R, Prévost P, Ivanenko Y, Berthoz A: Eye-head coordination

- for the steering of locomotion in humans: an anticipatory synergy. Neurosci Lett 1998, 253:115-118.

Head and gaze orientation were studied in subjects walking along $90^{\circ}$ corner trajectories both in the light and with the eyes closed. The head and eyes systematically deviated toward the future direction of the curved trajectory in anticipation of the turn of the trunk and feet

13. Grasso R, Assaiante C, Prévost P, Berthoz A: Development of anticipatory orienting strategies during locomotor tasks in children. Neurosci Biobehav Rev 1998, 22:533-539

Reports that anticipatory head movements toward the direction to be walked develop very early in childhood.

14. Berthoz A, Amorim A, Glasauer S, Grasso R, Takei Y, Viaud-Delmon I:

- Dissociation between distance and direction during locomotor navigation. In Wayfinding Behavior. Edited by Golledge RG. Baltimore: John Hopkins University Press; 1999:328-348.

This paper summarises several studies concerning the steering of locomotion. Subjects were asked to walk blindfolded around previously-seen circles or triangular paths. The experiments were performed both with normal subjects and patients with bilateral or unilateral vestibular lesions. Head movement was measured with computerised video techniques. The results suggest that there is a dissociation between the control of distance and direction. A similar hypothesis has been proposed for arm movements by others. The possible role of a newly-discovered head-direction cell system in the coding of direction is discussed.

15. McNaughton N: Cognitive dysfunction resulting from hippocampal hyperactivity - a possible cause of anxiety disorder? Pharmacol Biochem Behav 1997, 56:603-611.

16. Reiman EM: The application of positron emission tomography to the study of normal and pathologic emotions. J Clin Psychiatry 1997, 58(Suppl 16):4-12.

17. Jones RB, Humphris G, Lewis T: Do agoraphobics interpret the environment in large shops and supermarkets differently? $\mathrm{Br} \mathrm{J}$ Clin Psychol 1996, 35:635-637.

18. Kallai J, Koczan G, Szabo I, Molnar P, Varga J: An experimental study to operationally define and measure spatial orientation in panic agoraphobic subjects, generalized anxiety and healthy control groups. Behav Cogn Psychother 1995, 23:145-152.
19. JacobRG, Moeller MB, Turner SM, WallC: Otoneurological examination in panic disorder and agoraphobia with panic attacks: a pilot study. Am J Psychiatry 1985, 142:715-720.

20. Yardley L, Burgneay J, Nazareth I, Luxon L: Neuro-otological and

-. psychiatric abnormalities in a community sample of people with dizziness: a blind, controlled investigation. J Neurol Neurosurg Psychiatry 1998, 65:679-84.

This study determines the extent of neuro-otological and psychiatric abnormalities associated with dizziness among 2064 working-age adults by means of postal questionnaires. A careful description of co-morbidity between dizziness, anxiety and avoidance behaviour is provided. More than half of patients reporting anxiety also complained of dizziness, and the prevalence of reported anxiety or panic-related symptoms was three times greater among people with dizziness than among those without. The results suggest that this association could arise from several processes, and underline the importance of investigating the neurophysiological mechanisms involved in the interrelation between physiological and psychological manifestations.

21. Asmundson GJ, Larsen DK, Stein MB: Panic disorder and vestibular - disturbance: an overview of empirical findings and clinical implications. J Psychosom Res 1998, 44:107-120.

The authors provide an extensive overview of the literature on vestibular function in patients with panic disorder, and on the evaluation of panic symptomatology in patients with vestibular complaints. They discuss clinical implications suggested by the findings, and outline the unique and reciprocal relationship between balance problems and anxiety disorders.

22. Simon NM, Pollack MH, Tuby KS, Stern TA: Dizziness and panic

- disorder: a review of the association between vestibular dysfunction and anxiety. Ann Clin Psychiatry 1998, 10:75-80.

Articles from 1990-1997 that addressed the association of panic disorder and/or agoraphobia with vestibular dysfunction are reviewed and discussed This work emphasises the lack of uniformity in diagnostics or definitions of vestibular dysfunction, and concludes that it is unclear if reported vestibular abnormalities are specific to panic disorder.

23. Marks I: Space 'phobia': a pseudo-agoraphobic syndrome J Neurol Neurosurg Psychiatry 1981, 44:387-391.

24. Jacob RG, Lilienfield SO, Furman JMR, Durrant JD, Turner SM: Panic disorder with vestibular dysfunction: further clinical observations and description of space and motion phobic stimuli. J Anxiety Disord 1989, 3:117-130.

25. Brandt T, Dieterich M: Phobischer attacken-schwankschwindel, ein neues syndrom? Münch Med Wochenschr 1986, 128:247-250. [Title translation: Phobic vertigo attack: a new syndrome?]

26. Page NG, Gresty MA: Motorist's vestibular disorientation syndrome. J Neurol Neurosurg Psychiatry 1985, 48:729-735.

27. Bronstein AM: Visual vertigo syndrome: clinical and posturography findings. J Neurol Neurosurg Psychiatry 1995, 59:472-476.

28. Yardley L, Britton J, Lear S, Bird J, Luxon LM: Relationship between balance system function and agoraphobic avoidance. Behav Res Ther 1995, 33:435-439.

29. Jacob RG, Furman JMR, Durrant JD, Turner SM: Panic, agoraphobia, and vestibular dysfunction. Am J Psychiatry 1996, 153:503-512.

30. Jacob RG, Furman JM, Durrant JD, Turner SM: Surface dependence: a balance control strategy in panic disorder with agoraphobia. Psychosom Med 1997, 59:323-330.

31. Viaud-Delmon I, Siegler I, Israël I, Jouvent R, Berthoz A: Eye deviation during rotation in darkness in trait anxiety: An early expression of perceptual avoidance? Biol Psychiatry 2000, 47(2):in press.

32. Darlington CL, Smith PF: Further evidence for gender differences in circular vection. J Vestib Res 1998, 8:151-153.

33. Lawther A, Griffin MJ: Prediction of the incidence of motion sickness from the magnitude, frequency, and duration of vertical oscillation. J Acoust Soc Am 1987, 82:957-966.

34. Murphy TP: Mal de debarquement syndrome: a forgotten entity? Otolaryngol Head Neck Surg 1993, 109:10-13.

35. Viaud-Delmon I, Ivanenko YP, Berthoz A, Jouvent R: Sex, lies and virtual reality. Nat Neurosci 1998, 1:15-16.

The change in vestibular perception of rotation tested in darkness was greater in males than in females after exposure to sensorial conflict. This conflict was produced by the rotation of the subject for $45 \mathrm{~min}$ around an earth-vertical axis while immersed in a virtual environment in which the visual world was moving at a different speed from the head rotation. Vestibular perception of rotation was tested before and after exposure to the conflicting signals. 
36. Maguire EA, Burgess N, O'Keefe: Human spatial navigation: cognitive maps, sexual dimorphism, and neural substrates. Curr Opin Neurobiol 1999, 9:171-177.

37. McEwen BS, Alves SE, Bulloch K, Weiland NG: Clinically relevan basic science studies of gender differences and sex hormone effects. Psychopharmacol Bull 1998, 34:251-259.

38. Rolls ET, Robertson, RG, Georges-François, P: Spatial view cells in the primate hippocampus. Eur J Neurosci 1997, 9:1789-1794.

39. Siegler I, Israël I, Berthoz A: Shift of the beating field of vestibular - nystagmus: an orientation strategy? Neurosci Lett 1998, 254:93-96. This study investigated in humans whether the shift of the beating field observed during vestibular nystagmus could be related to some strategy of orientation. It suggests that this shift is the manifestation of two different orientation strategies based on allocentric or egocentric reference frames. Subjects who preferably used the first strategy exhibited large shifts of the beating field, whereas those selecting the second did not exhibit any shift.

40. Amorim MA, Glasauer S, Corpinot K, Berthoz A: Updating an object's orientation and location during non visual navigation: a comparison between two processing modes. Percept Psychophys 1997, 59:404-418.

41. Ghaem O, Mellet E, Crivello F, Tzourio N, Mazoyer B, Berthoz A, Denis $\mathrm{M}$ : Mental navigation along memorized routes activates the hippocampus, precuneus and insula. Neuroreport 1997, 8.739-744. 